This is an electronic reprint of the original article. This reprint may differ from the original in pagination and typographic detail.

Author(s): Almeida, A.; Harjulehto, P.; Hästö, P.; Lukkari, Teemu

Title: $\quad$ Riesz and Wolff potentials and elliptic equations in variable exponent weak Lebesgue spaces

Year: $\quad 2015$

Version:

Please cite the original version:

Almeida, A., Harjulehto, P., Hästö, P., \& Lukkari, T. (2015). Riesz and Wolff potentials and elliptic equations in variable exponent weak Lebesgue spaces. Annali di Matematica Pura ed Applicata, 94(2), 405-424. https://doi.org/10.1007/s10231-0130382-2

All material supplied via JYX is protected by copyright and other intellectual property rights, and duplication or sale of all or part of any of the repository collections is not permitted, except that material may be duplicated by you for your research use or educational purposes in electronic or print form. You must obtain permission for any other use. Electronic or print copies may not be offered, whether for sale or otherwise to anyone who is not an authorised user. 


\title{
RIESZ AND WOLFF POTENTIALS AND ELLIPTIC EQUATIONS IN VARIABLE EXPONENT WEAK LEBESGUE SPACES
}

\author{
A. ALMEIDA, P. HARJULEHTO, P. HÄSTÖ AND T. LUKKARI
}

\begin{abstract}
We prove optimal integrability results for solutions of the $p(\cdot)$-Laplace equation in the scale of (weak) Lebesgue spaces. To obtain this, we show that variable exponent Riesz and Wolff potentials map $L^{1}$ to variable exponent weak Lebesgue spaces.
\end{abstract}

\section{INTRODUCTION}

In this paper we study the mapping properties of variable exponent Riesz and Wolff potentials on weak $L^{p(\cdot)}$ spaces, denoted by $w-L^{p(\cdot)}$. Our interest stems mainly from the following problem, whose solution is presented in Section 8. Consider appropriately defined weak solutions to the boundary value problem

$$
\begin{cases}-\operatorname{div}\left(|\nabla u|^{p(x)-2} \nabla u\right)=f & \text { in } \Omega, \\ u=0 & \text { on } \partial \Omega\end{cases}
$$

when the data $f$ is merely an $L^{1}$ function. We refer to [14] for an extensive survey of such equations with non-standard growth. Based on the constant exponent case and computations on explicit solutions, one expects in the $L^{1}$-situation that

$$
u \in w-L_{\mathrm{loc}}^{\frac{n}{n-p(\cdot)}(p(\cdot)-1)}(\Omega) \quad \text { and } \quad|\nabla u| \in w-L_{\text {loc }}^{\frac{n}{n-1}(p(\cdot)-1)}(\Omega) .
$$

By earlier results of Sanchón and Urbano [28, Remark 3.3], the gradient belongs to the space $w-L_{\text {loc }}^{\frac{n(p(\cdot)-1)}{n-1}-\varepsilon}(\Omega)$, while Bögelein and Habermann [6] proved that it is in $L_{\mathrm{loc}}^{\frac{n(p(\cdot)-1)}{n-1}-\varepsilon}(\Omega)$, for any $\varepsilon>0$. By elementary properties of weak spaces (Proposition 3.4) these two results are in fact equivalent. However, as (1.2) is the borderline case $\varepsilon=0$, it has turned out to be hard to reach. As in the constant exponent case, when $\varepsilon=0$ the inclusions into the (strong) Lebesgue space do not hold.

Our approach to this problem relies on the recent pointwise potential estimates for solutions and their gradients to problems with $L^{1}$ or measure data, see $[11,12,25]$. The case of equations similar to (1.1) is covered

Date: September 1, 2015.

2000 Mathematics Subject Classification. 47H99 (46B70, 46E30, 35J60, 31C45).

Key words and phrases. Variable exponent, weak Lebesgue space, real interpolation, Riesz potential, Wolff potential, non-standard growth condition, integrability of solutions, integrability estimates. 
in [6]. The potential that appears in the nonlinear situation is the Wolff potential, given by

$$
\mathcal{W}_{\alpha, p}^{f}(x):=\int_{0}^{\infty}\left(\frac{\int_{B(x, r)}|f(y)| d y}{r^{n-\alpha p}}\right)^{1 /(p-1)} \frac{d r}{r}
$$

At a given point $x$, a solution to (1.1) is controlled by $\mathcal{W}_{1, p(x)}^{f}(x)$, and its gradient is controlled by $\mathcal{W}_{1 / p(x), p(x)}^{f}(x)$.

These estimates are the nonlinear counterparts of representation formulas, as properties of solutions may be deduced from the properties of the potentials. Our aim is to exploit this, and establish a local version of (1.2) by proving that the Wolff potential $\mathcal{W}_{\alpha(x), p(x)}^{f}(x)$ has the appropriate mapping properties. This answers the open problem posed by Sanchón and Urbano [28, Remark 3.3] and completes the generalization of the Wolff-potential approach for (1.1) started by Bögelein and Habermann in [6].

The usual way to look at the mapping properties of the Wolff potential is to estimate it pointwise by the Havin-Maz'ya potential (see [15]), which is an iterated Riesz potential. Thus we study the mapping properties of the Riesz potential as well. For (strong) Lebesgue spaces these properties are well known, see [8, 26, 27] and [9, Section 6.1]. Here we deal with the novel case of weak Lebesgue spaces.

Our first result, Theorem 4.3, is the strong-to-weak estimate for the Riesz

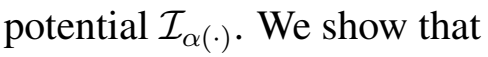

$$
\mathcal{I}_{\alpha(\cdot)}: L^{r(\cdot)}(\Omega) \rightarrow w-L^{r_{\alpha}^{\#}(\cdot)}(\Omega),
$$

where $\Omega$ is an open, bounded set in $\mathbb{R}^{n}$, the target space is a weak variable exponent Lebesgue space and $r_{\alpha}^{\#}:=n r /(n-\alpha r)$ is the (pointwise) Sobolev conjugate of $r$. For $r^{-}:=\inf r>1$, strong-to-strong boundedness has been known for ten years [8], so the novelty lies in the inclusion of the case $r^{-}=1$. In contrast to the constant exponent case, this is not enough for us; surprisingly, the fact that

$$
f \in L^{r(\cdot)}(\Omega) \quad \Longrightarrow \quad\left(\mathcal{I}_{\alpha(\cdot)} f\right)^{q(\cdot)} \in w-L^{\frac{r_{\alpha}^{\#}(\cdot)}{q(\cdot)}}(\Omega)
$$

for every log-Hölder continuous positive function $q$ requires a separate proof. This proof is based on pointwise estimate between the Riesz potential and the Hardy-Littlewood maximal operator.

Then we study how the Riesz potential acts on weak Lebesgue spaces, as this situation will inevitably happen when dealing with the Wolff potential on $L^{1}$. This turns out to be a difficult question because the weak Lebesgue spaces are not well-behaved. We show that the weak Lebesgue space is an interpolation space (Theorem 5.1). This allows us to use real interpolation to get weak-to-weak boundedness of the maximal operator: 
Theorem 1.3. Let $p$ be a bounded measurable function with $p^{-}>1$. If $\mathcal{M}: L^{p(\cdot)}\left(\mathbb{R}^{n}\right) \rightarrow L^{p(\cdot)}\left(\mathbb{R}^{n}\right)$ is bounded, then so is $\mathcal{M}: w-L^{p(\cdot)}\left(\mathbb{R}^{n}\right) \rightarrow$ $w-L^{p(\cdot)}\left(\mathbb{R}^{n}\right)$.

In particular, $\mathcal{M}: w-L^{p(\cdot)}\left(\mathbb{R}^{n}\right) \rightarrow w-L^{p(\cdot)}\left(\mathbb{R}^{n}\right)$ is bounded when $p$ is $\log$-Hölder continuous and $p^{-}>1$.

With a complicated application of Hedberg's trick, we then prove in Theorem 6.6 that

$f \in w-L^{q(\cdot)}(\Omega)$ and $|f|^{q(\cdot) / q_{\alpha}^{\#}(\cdot)} \in w-L^{q_{\alpha}^{\#}(\cdot)}(\Omega) \quad \Longrightarrow \quad \mathcal{I}_{\alpha(\cdot)} f \in w-L^{q_{\alpha}^{\#}(\cdot)}(\Omega)$.

We combine these results, and obtain in Theorem 7.2 that

$$
f \in L^{1}(\Omega) \quad \Longrightarrow \quad \mathcal{W}_{\alpha(x), p(x)}^{f} \in w-L^{\frac{n(p(\cdot)-1)}{n-\alpha(\cdot) p(\cdot)}}(\Omega) .
$$

A combination of (1.4) and the pointwise potential estimates now yields (1.2), provided that an appropriate notion of solutions to (1.1) is used. This requires some care, as $L^{1}(\Omega)$ is not contained in the dual of the natural Sobolev space $W_{0}^{1, p(\cdot)}(\Omega)$. Here we use the notion of solutions obtained as limits of approximations, or SOLAs for short. The idea is to approximate $f$ with more regular functions, prove uniform a priori estimates in a larger Sobolev space $W_{0}^{1, q(\cdot)}(\Omega)$, and then pass to the limit by compactness arguments. This way, one finds a function $u \in W_{0}^{1, q(\cdot)}(\Omega)$ such that (1.1) holds in the sense of distributions. See e.g. $[4,5,18]$ for a few implementations of this basic idea, and $[21,28]$ for equations similar to the $p(\cdot)$-Laplacian. In fact, the same approximation approach is used in proving the potential estimates.

A representative special case of what comes out by combining nonlinear potential estimates and our results about the Wolff potential is the following theorem.

Theorem 1.5. Let $f \in L^{1}(\Omega)$, and let $p$ be bounded and Hölder continuous with $p^{-} \geqslant 2$. Suppose that $u$ is a SOLA to (1.1). Then

$$
u \in w-L_{\mathrm{loc}}^{\frac{n(p(\cdot)-1)}{n \cdot p(\cdot)}}(\Omega) \quad \text { and } \quad|\nabla u| \in w-L_{\mathrm{loc}}^{\frac{n(p(\cdot)-1)}{n-1}}(\Omega) .
$$

In other words, (1.2) holds locally under suitable assumptions. Similar results also follow for the fundamental objects of nonlinear potential theory, the $p(\cdot)$-superharmonic functions. Finally, by examining the counterpart of the fundamental solution (Example 8.14) we show that the exponents in Theorem 1.5 are sharp, as expected.

\section{NotATION}

We write simply $A \lesssim B$ if there is a constant $c$ such that $A \leqslant c B$. We also use the notation $A \approx B$ when $A \lesssim B$ and $A \gtrsim B$. For compatible vector spaces, the space $X \cap Y$ is defined by the norm $\|f\|:=\max \left\{\|f\|_{X},\|f\|_{Y}\right\}$ while $X+Y$ is defined by $\|f\|:=\inf _{f_{1}+f_{2}=f}\left\|f_{1}\right\|_{X}+\left\|f_{2}\right\|_{Y}$. 
Let $U \subset \mathbb{R}^{n}$. For $g: U \rightarrow \mathbb{R}$ and $A \subset U$ we denote

$$
g_{A}^{+}:=\underset{x \in A}{\operatorname{essipup}} g(x) \quad \text { and } \quad g_{A}^{-}:=\operatorname{essinf}_{x \in A} g(x)
$$

and abbreviate $g^{+}:=g_{U}^{+}$and $g^{-}:=g_{U}^{-}$. We say that $g: U \rightarrow \mathbb{R}$ satisfies the local log-Hölder continuity condition if

$$
|g(x)-g(y)| \leqslant \frac{c}{\log (e+1 /|x-y|)}
$$

for all $x, y \in U$. We will often use the fact that $g$ is locally log-Hölder continuous if and only if

$$
|B|^{g_{B}^{-}-g_{B}^{+}} \lesssim 1
$$

for all balls $B \cap U \neq \emptyset$. If

$$
\left|g(x)-g_{\infty}\right| \leqslant \frac{c^{\prime}}{\log (e+|x|)}
$$

for some $g_{\infty} \geqslant 1, c^{\prime}>0$ and all $x \in U$, then we say $g$ satisfies the logHölder decay condition (at infinity). If both conditions are satisfied, we simply speak of log-Hölder continuity. By the log-Hölder constant we mean $\max \left\{c, c^{\prime}\right\}$.

By a variable exponent we mean a measurable function $p: U \rightarrow(0, \infty)$ such that $0<p^{-} \leqslant p^{+}<\infty$. The set of variable exponents is denoted by $\mathcal{P}_{0}(U) ; \mathcal{P}_{1}(U)$ is the subclass with $1 \leqslant p^{-}$. By $\mathcal{P}_{0}^{\log }(U)$ and $\mathcal{P}_{1}^{\log }(U)$ we denote the respective subsets consisting of log-Hölder continuous exponents.

We define a modular on the set of measurable functions by setting

$$
\varrho_{L^{p(\cdot)}(U)}(f):=\int_{U}|f(x)|^{p(x)} d x .
$$

The variable exponent Lebesgue space $L^{p(\cdot)}(U)$ consists of all the measurable functions $f: U \rightarrow \mathbb{R}$ for which the modular $\varrho_{L^{p(\cdot)}(U)}(f)$ is finite. The Luxemburg norm on this space is defined as

$$
\|f\|_{L^{p(\cdot)}(U)}:=\inf \left\{\lambda>0: \varrho_{L^{p(\cdot)}(U)}\left(\frac{f}{\lambda}\right) \leqslant 1\right\} .
$$

Equipped with this norm, $L^{p(\cdot)}(U)$ is a Banach space. We use the abbreviation $\|f\|_{p(\cdot)}$ to denote the norm in the whole space under consideration. The norm and the modular are related by the inequalities

$$
\min \left\{\|f\|_{L^{p(\cdot)}(U)}^{p^{+}},\|f\|_{L^{p(\cdot)}(U)}^{p^{-}}\right\} \leqslant \varrho_{L^{p(\cdot)}(U)}(f) \leqslant \max \left\{\|f\|_{L^{p(\cdot)}(U)}^{p^{+}},\|f\|_{L^{p(\cdot)}(U)}^{p^{-}}\right\} .
$$

For open sets $U$, the variable exponent Sobolev space $W^{1, p(\cdot)}(U)$ consists of functions $u \in L^{p(\cdot)}(U)$ whose distributional gradient $\nabla u$ belongs to $L^{p(\cdot)}(U)$. The norm

$$
\|u\|_{W^{1, p(\cdot)(U)}}:=\|u\|_{L^{p(\cdot)(U)}}+\|\nabla u\|_{L^{p(\cdot)}(U)}
$$

makes $W^{1, p(\cdot)}(U)$ a Banach space. The Sobolev space with zero boundary values, denoted by $W_{0}^{1, p(\cdot)}(U)$, is the completion of $C_{0}^{\infty}(U)$ with respect to 
the norm of $W^{1, p(\cdot)}(U)$. This definition does not cause any difficulties: the assumptions on $p$ in Section 8, where we use Sobolev spaces, are enough to guarantee that smooth functions are dense in the Sobolev space.

More information and proofs for the above facts can be found for example from [9, Chapters 2, 4, 8, and 9].

By $\Omega$ we always denote an open bounded set in $\mathbb{R}^{n}$.

In auxiliary results we use the convention that constants (implicit or explicit) depend on the assumptions stated in the result. For instance, in Proposition 3.4 the assumptions are that $p, q \in \mathcal{P}_{0}(\Omega)$ and $(p-q)^{-}>0$, so in this case, the implicit constant (potentially) depends on $p^{-}, p^{+}, q^{-}, q^{+}$, $(p-q)^{-}$, and on the dimension $n$.

\section{BASIC PROPERTIES OF WEAK LEBESGUE SPACES}

Definition 3.1. Let $A \subset \mathbb{R}^{n}$ be measurable. A measurable function $f$ : $A \rightarrow \mathbb{R}$ belongs to the weak Lebesgue space $w-L^{p(\cdot)}(A)$ if

$$
\|f\|_{w-L^{p(\cdot)}(A)}:=\sup _{\lambda>0} \lambda\left\|\chi_{\{|f|>\lambda\}}\right\|_{L^{p(\cdot)}(A)}<\infty .
$$

The inequalities (2.2) imply that the requirement in Definition 3.1 is equivalent with

$$
\sup _{\lambda>0} \int_{\{|f|>\lambda\}} \lambda^{p(x)} d x<\infty .
$$

Another immediate consequence of (2.2) which we will use in the proofs below is that

$$
\|f\|_{w-L^{p(\cdot)}(A)} \leqslant 1 \quad \text { if and only if } \sup _{\lambda>0} \int_{\{|f|>\lambda\}} \lambda^{p(x)} d x \leqslant 1 .
$$

We immediately obtain the following two inclusions:

- $L^{p(\cdot)}\left(\mathbb{R}^{n}\right) \subset w-L^{p(\cdot)}\left(\mathbb{R}^{n}\right)$, since $\lambda \chi_{\{|f|>\lambda\}} \leqslant|f|$;

- for bounded sets, $w-L^{p(\cdot)}(\Omega) \subset w-L^{q(\cdot)}(\Omega)$ when $p \geqslant q$, since the inequality $\|\cdot\|_{p(\cdot)} \gtrsim\|\cdot\|_{q(\cdot)}$ holds for the corresponding strong spaces.

The following result is from [28, Proposition 2.5]. We present a simpler proof here.

Proposition 3.4. Let $p, q \in \mathcal{P}_{0}(\Omega)$. If $(p-q)^{-}>0$, then $w$ - $L^{p(\cdot)}(\Omega) \subset$ $L^{q(\cdot)}(\Omega)$. 
Proof. Let $f \in w-L^{p(\cdot)}(\Omega)$. We write $E_{i}:=\left\{2^{i} \leqslant|f|<2^{i+1}\right\}$ for every $i=0,1,2, \ldots$ Then $\Omega=\bigcup_{i=0}^{\infty} E_{i} \cup\{|f|<1\}$. We obtain

$$
\begin{aligned}
\int_{\Omega}|f|^{q(x)} d x & \leqslant \sum_{i=0}^{\infty} \int_{E_{i}} 2^{(i+1) q(x)} d x+|\Omega| \\
& \leqslant 2^{q^{+}} \sum_{i=0}^{\infty} \int_{\left\{|f| \geqslant 2^{i}\right\}} 2^{i p(x)} 2^{-i(p(x)-q(x))} d x+|\Omega| \\
& \leqslant 2^{q^{+}} \sum_{i=0}^{\infty} 2^{-i(p-q)^{-}} \int_{\left\{|f| \geqslant 2^{i}\right\}} 2^{i p(x)} d x+|\Omega| \\
& \leqslant 2^{q^{+}} \max \left\{\|f\|_{w-L^{p(\cdot)}(\Omega)}^{p^{+}},\|f\|_{w-L^{p(\cdot)}(\Omega)}^{p^{-}}\right\} \sum_{i=0}^{\infty} 2^{-i(p-q)^{-}}+|\Omega|<\infty .
\end{aligned}
$$

Note that Proposition 3.4 works not only for bounded sets but also for every open set with a finite measure. It can be similarly proved that

$$
w-L^{p(\cdot)}\left(\mathbb{R}^{n}\right) \subset L^{q(\cdot)}\left(\mathbb{R}^{n}\right)+L^{r(\cdot)}\left(\mathbb{R}^{n}\right)
$$

for all exponents $p, q, r$ with $(p-q)^{-}>0$ and $r \geqslant p$.

It is easy to show that $f \in L^{p(\cdot)}\left(\mathbb{R}^{n}\right)$ if and only if $|f|^{q(\cdot)} \in L^{\frac{p(\cdot)}{q(\cdot)}}\left(\mathbb{R}^{n}\right)$. However, the same is not true for the weak Lebesgue space. Indeed, in this case the following property holds:

Proposition 3.5. Let $p \in \mathcal{P}_{0}\left(\mathbb{R}^{n}\right)$. Then $|f|^{q(\cdot)} \in w-L^{\frac{p(\cdot)}{q(\cdot)}}\left(\mathbb{R}^{n}\right)$ for every function $q: \mathbb{R}^{n} \rightarrow(0, \infty)$ if and only if $f \in L^{p(\cdot)}\left(\mathbb{R}^{n}\right)$.

If $q$ is constant, then $f \in w-L^{p(\cdot)}\left(\mathbb{R}^{n}\right)$ if and only if $|f|^{q} \in w-L^{\frac{p(\cdot)}{q}}\left(\mathbb{R}^{n}\right)$.

Proof. If $f \in L^{p(\cdot)}\left(\mathbb{R}^{n}\right)$, then

$$
\varrho_{\frac{p(\cdot)}{q(\cdot)}}\left(\lambda \chi_{\left\{|f|^{q(\cdot)}>\lambda\right\}}\right) \leqslant \varrho_{\frac{p(\cdot)}{q(\cdot)}}\left(|f|^{q(\cdot)}\right)=\varrho_{p(\cdot)}(f),
$$

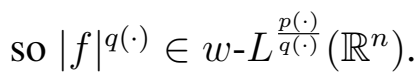

Conversely, let $f$ be such that $|f|^{q(\cdot)} \in w-L^{\frac{p(\cdot)}{q(\cdot)}}\left(\mathbb{R}^{n}\right)$ for every function $q: \mathbb{R}^{n} \rightarrow(0, \infty)$. Define $q: \mathbb{R}^{n} \rightarrow(0, \infty)$ such that

$$
\left(\frac{1}{2}\right)^{\frac{1}{q(x)}}=\frac{1}{2} \min \{|f(x)|, 1\}
$$

for $|f(x)|>0$ and set $q=1$ in $\{f=0\}$. Let $\lambda=\frac{1}{2}$ and note that $\left\{|f|^{q(\cdot)}>\lambda\right\}=\{|f|>0\}$. Then we find that

$$
\begin{aligned}
\varrho_{\frac{p(\cdot)}{q(\cdot)}}\left(\lambda \chi_{\left\{|f| f^{q(\cdot)}>\lambda\right\}}\right) & =\int_{\left\{|f|^{q(\cdot)}>\lambda\right\}} \lambda^{\frac{p(x)}{q(x)}} d x=\int_{\{|f|>0\}} 2^{-p(x)} \min \{|f|, 1\}^{p(x)} d x \\
& \geqslant 2^{-p^{+}} \int_{\mathbb{R}^{n}} \min \{|f|, 1\}^{p(x)} d x \geqslant 2^{-p^{+}} \varrho_{p(\cdot)}\left(f \chi_{\{|f| \leqslant 1\}}\right) .
\end{aligned}
$$


Hence by the definition of the weak space we obtain that $\varrho_{p(\cdot)}\left(f \chi_{\{|f| \leqslant 1\}}\right)$ is finite.

To estimate large values of $f$, let $q: \mathbb{R}^{n} \rightarrow(0, \infty)$ be such that

$$
2^{\frac{1}{q(x)}}=\frac{1}{2} \max \{|f(x)|, 1\} .
$$

Let $\lambda=2$ and note that $\left\{|f|^{q(\cdot)}>\lambda\right\} \supset\{f \geqslant 1\}$. Now by a similar calculation as above, we conclude that $\varrho_{p(\cdot)}\left(f \chi_{\{|f| \geqslant 1\}}\right)$ is finite. Thus $f \in$ $L^{p(\cdot)}\left(\mathbb{R}^{n}\right)$.

The last claim, regarding the case of $q$ constant, follows from a change of variables:

$$
\left(\sup _{\lambda>0} \lambda\left\|\chi_{\{|f|>\lambda\}}\right\|_{p(\cdot)}\right)^{q}=\sup _{\lambda>0} \lambda\left\|\chi_{\left\{|f|>\lambda^{\frac{1}{q}}\right\}}\right\|_{p(\cdot)}^{q}=\sup _{\lambda>0} \lambda\left\|\chi_{\{|f| q>\lambda\}}\right\|_{\frac{p(\cdot)}{q}} .
$$

\section{STRONG-TO-WEAK ESTIMATES FOR THE RIESZ POTENTIAL}

Let $\alpha: \Omega \rightarrow \mathbb{R}$ be log-Hölder continuous with $0<\alpha^{-} \leqslant \alpha^{+}<n$. We consider the Riesz potential

$$
\mathcal{I}_{\alpha(\cdot)} f(x):=\int_{\Omega} \frac{|f(y)|}{|x-y|^{n-\alpha(y)}} d y
$$

in $\Omega$, and write

$$
p_{\alpha}^{\#}(x):=\frac{n p(x)}{n-\alpha(x) p(x)} .
$$

Because $\Omega$ is bounded and $\alpha$ is log-Hölder continuous we observe as in [16, p. 270] that $\mathcal{I}_{\alpha(\cdot)} f(x)$ and

$$
\mathcal{I}_{\alpha(x)} f(x)=\int_{\Omega} \frac{|f(y)|}{|x-y|^{n-\alpha(x)}} d y
$$

are pointwise equivalent. Thus we obtain the following result from [9, Proposition 6.1.6].

Proposition 4.1. Let $p \in \mathcal{P}_{1}^{\log }(\Omega), \alpha \in \mathcal{P}_{0}^{\log }(\Omega)$ and $(\alpha p)^{+}<n$. Then

$$
\mathcal{I}_{\alpha(\cdot)} f(x) \lesssim[\mathcal{M} f(x)]^{1-\frac{\alpha(x) p(x)}{n}} .
$$

for every $f \in L^{p(\cdot)}(\Omega)$ with $\|f\|_{p(\cdot)} \leqslant 1$.

Here $\mathcal{M}$ denotes the Hardy-Littlewood maximal function given by

$$
\mathcal{M} f(x):=\sup _{t>0}|f|_{B(x, t)}:=\sup _{t>0} \frac{1}{|B(x, t)|} \int_{B(x, t)}|f(y)| d y .
$$

For a measurable function $f$ and measurable set $B$ we use the notation $f_{B}$ for the mean integral of $f$ over $B$.

We also need the following Jensen-type inequality. The lemma is a restatement of [9, Theorem 4.2.4] in our current notation, cf. also the proof of Lemma 4.3.6 in the same source. 
Lemma 4.2. Let $A \subset \mathbb{R}^{n}$ be measurable and $p \in \mathcal{P}_{1}^{\log }(A)$. If $f \in L^{p(\cdot)}(A)$ and $\|f\|_{p(\cdot)} \leqslant 1$, then

$$
\left(|f|_{B}\right)^{p(x)} \lesssim\left(|f|^{p(\cdot)}+h\right)_{B}
$$

for every $x \in A$ and every ball $B \subset A$ containing $x$, where $h \in w-L^{1}(A) \cap$ $L^{\infty}(A)$.

The next statement shows that the Riesz potentials behave as expected in the variable exponent weak space. We will use the exponent $q$ to overcome the difficulty illustrated in Proposition 3.5.

Theorem 4.3. Suppose that $p \in \mathcal{P}_{1}^{\log }(\Omega), \alpha \in \mathcal{P}_{0}^{\log }(\Omega)$ and $(\alpha p)^{+}<n$. If $f \in L^{p(\cdot)}(\Omega)$, then $\left(\mathcal{I}_{\alpha(\cdot)} f\right)^{q(\cdot)} \in w-L^{p_{\alpha}^{\#}(\cdot) / q(\cdot)}(\Omega)$ for every $q \in \mathcal{P}_{0}^{\log }(\Omega)$.

Proof. By (3.2), it is enough to show that for every $f \in L^{p(\cdot)}(\Omega)$ with $\|f\|_{p(\cdot)} \leqslant 1$ and every $t>0$ we have

$$
\int_{\left\{\left(\mathcal{I}_{\alpha(\cdot)} f\right)^{q(\cdot)}>t\right\}} t^{p_{\alpha}^{\#}(x) / q(x)} d x \lesssim 1 .
$$

By Proposition 4.1, for a suitable $c>0$,

$$
\left\{\left(\mathcal{I}_{\alpha(\cdot)} f(x)\right)^{q(x)}>t\right\} \subset\left\{c[\mathcal{M} f(x)]^{\frac{p(x) q(x)}{p_{\alpha}^{\#}(x)}}>t\right\}=: E .
$$

By the definition of the maximal function, for every $x \in E$ we may choose $B_{x}:=B\left(x, r_{x}\right)$ such that $c\left(|f|_{B_{x}}\right)^{\frac{p(x) q(x)}{p_{\alpha}^{\#}(x)}}>t$. Since $\|f\|_{1} \lesssim\|f\|_{p(\cdot)} \leqslant 1$ we get $|f|_{B_{x}} \lesssim\left|B_{x}\right|^{-1}$. Denote $r:=p q / p_{\alpha}^{\#}$. Then

$$
t \lesssim|f|_{B_{x}}^{r(x)} \leqslant\left(1+|f|_{B_{x}}\right)^{r(x)}=\left(1+|f|_{B_{x}}\right)^{r(y)}\left(1+|f|_{B_{x}}\right)^{r(x)-r(y)},
$$

where $y \in B_{x}$. If $r(x)-r(y) \leqslant 0$, then $\left(1+|f|_{B_{x}}\right)^{r(x)-r(y)} \leqslant 1$. If $r(x)-r(y)>0$, then we obtain by log-Hölder continuity (see (2.1)) that

$\left(1+|f|_{B_{x}}\right)^{r(x)-r(y)} \leqslant\left(1+\left|B_{x}\right|^{-1}\right)^{r(x)-r(y)} \leqslant 2^{p^{+} q^{+}}\left(1+\left|B_{x}\right|^{r(y)-r(x)}\right) \lesssim 1$.

Hence we have for every $y \in B_{x}$ that

$$
t \lesssim\left(1+|f|_{B_{x}}\right)^{r(y)} .
$$

By the Besicovitch covering theorem there is a countable covering subfamily $\left(B_{i}\right)$ of $\left\{B_{x}\right\}$ with bounded overlap. Thus we obtain by Lemma 4.2 that

$$
\begin{aligned}
\int_{E} t^{p_{\alpha}^{\#}(x) / q(x)} d x & \leqslant \sum_{i} \int_{B_{i}} t^{p_{\alpha}^{\#}(x) / q(x)} d x \lesssim \sum_{i} \int_{B_{i}}\left(1+|f|_{B_{i}}\right)^{p(x)} d x \\
& \lesssim \sum_{i}\left(\int_{B_{i}}|f(y)|^{p(y)}+h(y) d y+\left|B_{i}\right|\right) \lesssim 1
\end{aligned}
$$




\section{REAL INTERPOLATION AND WEAK LEBESGUE SPACES}

It is well known that real interpolation between the spaces $L^{p}$ and $L^{\infty}$ gives a weak Lebesgue space in the limiting situation when the second interpolation parameter equals $\infty$. We shall prove that the same holds in the variable exponent setting.

We recall that, for $0<\theta<1$ and $0<q \leqslant \infty$, the interpolation space $\left(A_{0}, A_{1}\right)_{\theta, q}$ is formed from compatible quasi-normed spaces $A_{0}$ and $A_{1}$ by defining a norm as follows. For $a \in A_{0}+A_{1}$ we set

$$
\|a\|_{\left(A_{0}, A_{1}\right)_{\theta, q}}:= \begin{cases}\left(\int_{0}^{\infty}\left[t^{-\theta} K(t, a)\right]^{q} \frac{d t}{t}\right)^{1 / q} & \text { when } q<\infty, \\ \sup _{t>0} t^{-\theta} K(t, a) & \text { when } q=\infty .\end{cases}
$$

Here the Peetre $K$-functional is given by

$$
K(t, a):=K\left(t, a ; A_{0}, A_{1}\right):=\inf _{\substack{a_{0}+a_{1}=a \\ a_{0} \in A_{0}, a_{1} \in A_{1}}}\left(\left\|a_{0}\right\|_{A_{0}}+t\left\|a_{1}\right\|_{A_{1}}\right), \quad t>0 .
$$

We saw in Proposition 3.5 that weak $L^{p(\cdot)}$-spaces are not very well behaved. Real interpolation in the variable exponent setting is even more challenging (cf. [3, 17]). Fortunately, we can get quite far with the following special case, whose proof already is quite complicated.

Theorem 5.1. Let $p \in \mathcal{P}_{0}\left(\mathbb{R}^{n}\right)$. For $\theta \in(0,1)$,

$$
\left(L^{(1-\theta) p(\cdot)}\left(\mathbb{R}^{n}\right), L^{\infty}\left(\mathbb{R}^{n}\right)\right)_{\theta, \infty}=w-L^{p(\cdot)}\left(\mathbb{R}^{n}\right) .
$$

Proof. Denote $p_{0}:=(1-\theta) p$ and $X:=\left(L^{p_{0}(\cdot)}\left(\mathbb{R}^{n}\right), L^{\infty}\left(\mathbb{R}^{n}\right)\right)_{\theta, \infty}$. Then by definition

$$
\|f\|_{X}=\sup _{t>0} t^{-\theta} \inf _{f_{0}+f_{1}=f}\left(\left\|f_{0}\right\|_{p_{0}(\cdot)}+t\left\|f_{1}\right\|_{\infty}\right) .
$$

We assume without loss of generality that $f, f_{0}, f_{1} \geqslant 0$.

We start by proving that $\|f\|_{X} \gtrsim\|f\|_{w-L^{p(\cdot)}}$. Let $\lambda>0$ be such that $\|f\|_{w-L^{p(\cdot)}}<2 \lambda\left\|\chi_{A}\right\|_{p(\cdot)}$ where $A:=\{f>\lambda\}$. Then it remains to prove the second of the inequalities

$$
\|f\|_{X} \geqslant\left\|\lambda \chi_{A}\right\|_{X} \geqslant\left\|\lambda \chi_{A}\right\|_{p(\cdot)} \gtrsim\|f\|_{w-L^{p(\cdot)} .}
$$

Suppose that $f_{0}+f_{1}=f$ and that $\left\|f_{1}\right\|_{\infty}=s$. Then we see that

$$
\inf _{f_{0}=f-f_{1}}\left(\left\|f_{0}\right\|_{p_{0}(\cdot)}+t\left\|f_{1}\right\|_{\infty}\right)=\|f-\min \{f, s\}\|_{p_{0}(\cdot)}+t s .
$$

Hence in the definition of $\|f\|_{X}$ we may take the infimum over $s>0$ and functions $f_{1}:=\min \{f, s\}, f_{0}:=f-f_{1}$. Thus we calculate

$$
\begin{aligned}
\left\|\chi_{A}\right\|_{X} & =\sup _{t>0} t^{-\theta} \inf _{s \in[0,1]}\left((1-s)\left\|\chi_{A}\right\|_{p_{0}(\cdot)}+t s\right) \\
& =\sup _{t>0} t^{-\theta} \min \left\{\left\|\chi_{A}\right\|_{p_{0}(\cdot)}, t\right\} \\
& =\left\|\chi_{A}\right\|_{p_{0}(\cdot)}^{1-\theta}=\left\|\chi_{A}\right\|_{p(\cdot)} .
\end{aligned}
$$


This completes the proof of the inequality $\|f\|_{X} \gtrsim\|f\|_{w-L^{p(\cdot)} \text {. }}$

We show next that $\|f\|_{X} \lesssim\|f\|_{w-L^{p(\cdot)}}$. By homogeneity, it suffices to consider the case where the right hand side equals one. Thus by (3.3) we can assume that

$$
1 \geqslant \int_{\{f>\lambda\}} \lambda^{p(x)} d x=\int_{\{f>\lambda\}} \lambda^{\frac{p_{0}(x)}{1-\theta}} d x=\int_{\left\{f>z^{1-\theta}\right\}} z^{p_{0}(x)} d x
$$

for every $\lambda>0$.

Since $f_{0}=f-\min \{f, s\}=\max \{f, s\}-s=\max \{f-s, 0\}$, we need to prove that

$$
\sup _{t>0} t^{-\theta} \inf _{s>0}\left(\|\max \{f-s, 0\}\|_{p_{0}(\cdot)}+t s\right) \lesssim 1 .
$$

We choose $s:=t^{\theta-1}$ so that $t^{-\theta} t s=1$. Thus it suffices to show that

$$
\left\|t^{-\theta} \max \left\{f-t^{\theta-1}, 0\right\}\right\|_{p_{0}(\cdot)} \lesssim 1
$$

for all $t>0$. We next note that $\max \left\{f-t^{\theta-1}, 0\right\} \leqslant f \chi_{\left\{f>z^{1-\theta}\right\}}$ with $z:=\frac{1}{t}$. Thus by (2.2), it suffices to show that

$$
\int_{\left\{f>z^{1-\theta}\right\}}\left(z^{\theta} f\right)^{p_{0}(x)} d x \lesssim 1
$$

for all $z>0$. It is enough to show that the inequality holds for all $z=2^{k_{0}}$, $k_{0} \in \mathbb{Z}$.

Define

$$
A_{k}:=\left\{x \in \mathbb{R}^{n} \mid f(x) \in\left(2^{k(1-\theta)}, 2^{(k+1)(1-\theta)}\right]\right\}, \quad k \in \mathbb{Z} .
$$

For $z=2^{k}$, we observe that $A_{k} \subset\left\{f>z^{1-\theta}\right\}$ and thus conclude from (5.2) that

$$
\int_{A_{k}} 2^{k p_{0}(x)} d x \leqslant 1
$$

Substituting $z=2^{k_{0}}$ in (5.3), we find that it is enough to prove that

$$
\sum_{k=k_{0}}^{\infty} \int_{A_{k}}\left(2^{k_{0} \theta} 2^{(k+1)(1-\theta)}\right)^{p_{0}(x)} d x \lesssim 1
$$

for all $k_{0} \in \mathbb{Z}$. So we estimate

$$
\int_{A_{k}}\left(2^{k_{0} \theta} 2^{(k+1)(1-\theta)}\right)^{p_{0}(x)} d x \leqslant\left(2^{\left(k_{0}-k\right) \theta}\right)^{p_{0}^{-}} \int_{A_{k}} 2^{k p_{0}(x)} d x \leqslant 2^{\left(k_{0}-k\right) \theta p_{0}^{-}} .
$$

Hence it follows that

$$
\sum_{k=k_{0}}^{\infty} \int_{A_{k}}\left(2^{k_{0} \theta} 2^{(k+1)(1-\theta)}\right)^{p_{0}(x)} d x \leqslant \sum_{k=k_{0}}^{\infty} 2^{\left(k_{0}-k\right) \theta p_{0}^{-}}=\frac{1}{1-2^{-\theta p_{0}^{-}}}<\infty,
$$

which is the required upper bound. 
The following feature is the main property of the the real interpolation method [30, Proposition 2.4.1]: If $T$ is a linear operator which is bounded from $X_{0}$ to $Y_{0}$ and from $X_{1}$ to $Y_{1}$, then $T$ is bounded from

$$
\left(X_{0}, X_{1}\right)_{\theta, q} \text { to }\left(Y_{0}, Y_{1}\right)_{\theta, q}
$$

for $\theta \in(0,1)$ and $q \in(0, \infty]$. If simple functions are dense in the spaces, then the claim holds also for sublinear operators (cf. [7, Theorem 1.5.11], or [10, Corollary A.5] for the variable exponent case; see also [2, Lemma 4.1] for a discussion in a general framework). This, together with Theorem 5.1 for $X_{0}=Y_{0}=L^{p(\cdot)}\left(\mathbb{R}^{n}\right)$ and $X_{1}=Y_{1}=L^{\infty}\left(\mathbb{R}^{n}\right)$ yields the following corollary.

Corollary 5.4. Assume that $T$ is sublinear, $T: L^{p(\cdot)}\left(\mathbb{R}^{n}\right) \rightarrow L^{p(\cdot)}\left(\mathbb{R}^{n}\right)$ is bounded, and $T: L^{\infty}\left(\mathbb{R}^{n}\right) \rightarrow L^{\infty}\left(\mathbb{R}^{n}\right)$ is bounded. Then $T: w-L^{\lambda p(\cdot)}\left(\mathbb{R}^{n}\right) \rightarrow$ $w-L^{\lambda p(\cdot)}\left(\mathbb{R}^{n}\right)$ is bounded for every $\lambda>1$.

L. Diening has shown that the boundedness of $\mathcal{M}: L^{p(\cdot)}\left(\mathbb{R}^{n}\right) \rightarrow L^{p(\cdot)}\left(\mathbb{R}^{n}\right)$ implies the boundedness of $\mathcal{M}: L^{s p(\cdot)}\left(\mathbb{R}^{n}\right) \rightarrow L^{s p(\cdot)}\left(\mathbb{R}^{n}\right)$ for some $s<1$ [9, Theorem 5.7.2]. Furthermore, it is known that the maximal operator is bounded on $L^{p(\cdot)}\left(\mathbb{R}^{n}\right)$ when $p \in \mathcal{P}_{1}^{\log }\left(\mathbb{R}^{n}\right)$ and $p^{-}>1$ [9, Theorem 4.3.8]. In view of the previous result these facts immediately imply Theorem 1.3.

\section{WEAK-TO-WEAK ESTIMATES FOR THE RIESZ POTENTIAL}

As usual, we denote by $p^{\prime}$ the Hölder conjugate exponent of $p$, taken in a point-wise sense, $1 / p(x)+1 / p^{\prime}(x)=1$. Following Diening (and [9]), for exponents we use the notation $p_{B}$ to denote the harmonic mean of $p$ over the measurable set $B$,

$$
p_{B}:=\left(f_{B} \frac{1}{p(x)} d x\right)^{-1} .
$$

The following claim is proved as part of the proof of [9, Lemma 6.1.5].

Lemma 6.1. Let $p \in \mathcal{P}_{1}^{\log }\left(\mathbb{R}^{n}\right)$ with $1<p^{-} \leqslant p^{+}<\frac{n}{\alpha}$ for $\alpha \in(0, n)$. Then

$$
\left\||x-\cdot|^{\alpha-n} \chi_{\mathbb{R}^{n} \backslash B}\right\|_{L^{p^{\prime}(\cdot)\left(\mathbb{R}^{n}\right)}} \approx|B|^{-\frac{1}{\left(p_{\alpha}^{\#}\right)_{B}}}
$$

where $B$ is a ball centered at $x \in \mathbb{R}^{n}$.

We next generalize this claim to slightly more general norms, which will appear below when we estimate in the dual of a weak Lebesgue space. We need the following auxiliary result.

Lemma 6.2. For $\alpha, \beta, \delta, t>0$,

$$
\inf _{R \in[\delta, \infty)}\left(\frac{\alpha}{\beta} R^{-\beta}+t\left(R^{\alpha}-\delta^{\alpha}\right)\right) \approx \min \left\{t^{\frac{\beta}{\alpha+\beta}}, \delta^{-\beta}\right\},
$$

and the infimum occurs at $R<1$ if and only ift $>1$ and $\delta<1$. 
Proof. Denote $f(R):=\frac{\alpha}{\beta} R^{-\beta}+t\left(R^{\alpha}-\delta^{\alpha}\right)$. Then $f^{\prime}(R)=-\alpha R^{-\beta-1}+$ t $\alpha R^{\alpha-1}$, which equals zero when $R=t^{-1 /(\alpha+\beta)}=: R_{0}$. This is a minimum in $(0, \infty)$, since $f \rightarrow \infty$ at 0 and $\infty$. When $R=R_{0} \geqslant \delta$, we estimate $0 \leqslant t\left(R^{\alpha}-\delta^{\alpha}\right) \leqslant t R^{\alpha}=R^{-\beta}$. Hence we conclude that $f\left(R_{0}\right) \approx R_{0}^{-\beta}=$ $t^{\beta /(\alpha+\beta)}$. Also note that the unconstrained minimum occurs for $R<1$ if and only if $t>1$.

However, if $R_{0}<\delta$, then the constrained minimum occurs at $\delta$, in which case $f(\delta)=\frac{\alpha}{\beta} \delta^{-\beta} \approx \delta^{-\beta}$. Hence the estimate of the minimum equals

$$
t^{\beta /(\alpha+\beta)} \chi_{\left\{t^{-1 /(\alpha+\beta)} \geqslant \delta\right\}}+\delta^{-\beta} \chi_{\left\{t^{-1 /(\alpha+\beta)}<\delta\right\}}=\min \left\{t^{\frac{\beta}{\alpha+\beta}}, \delta^{-\beta}\right\} .
$$

Lemma 6.3. Let $p \in \mathcal{P}_{1}^{\log }\left(\mathbb{R}^{n}\right)$ with $1<p^{-} \leqslant p^{+}<\frac{n}{\alpha}$ for $\alpha \in(0, n)$ and let $\theta>0$ be so small that the infimum of $r:=(1-\theta) p$ is greater than 1. Then

$$
\left\||x-\cdot|^{\alpha-n} \chi_{\mathbb{R}^{n} \backslash B}\right\|_{\left(L^{r^{\prime}(\cdot)}\left(\mathbb{R}^{n}\right), L^{1}\left(\mathbb{R}^{n}\right)\right)_{\theta, 1}} \approx|B|^{-\frac{1}{\left(p_{\alpha}^{\#}\right)_{B}}}
$$

where $B$ is a ball centered at $x \in \mathbb{R}^{n}$.

Proof. Let $B:=B(x, \delta)$ and denote $f(y):=|x-y|^{\alpha-n} \chi_{\mathbb{R}^{n} \backslash B}(y)$. By the definition of the interpolation norm,

$$
\|f\|_{\left(L^{\left.r^{\prime}(\cdot), L^{1}\right)_{\theta, 1}}\right.}=\int_{0}^{\infty} t^{-\theta} \inf _{f_{1}+f_{2}=f}\left(\left\|f_{1}\right\|_{r^{\prime}(\cdot)}+t\left\|f_{2}\right\|_{1}\right) \frac{d t}{t} .
$$

Suppose that $f_{1}+f_{2}=f$ and denote $A:=\left\{\left|f_{1}\right| \geqslant \frac{1}{2}|f|\right\}$. Then $\left|f_{1}\right| \geqslant$ $\frac{1}{2}|f| \chi_{A}$ and $\left|f_{2}\right| \geqslant \frac{1}{2}|f| \chi_{\mathbb{R}^{n} \backslash A}$, so that

$$
\left\|f_{1}\right\|_{r^{\prime}(\cdot)}+t\left\|f_{2}\right\|_{1} \geqslant \frac{1}{2}\left\|f \chi_{A}\right\|_{r^{\prime}(\cdot)}+\frac{1}{2} t\left\|f \chi_{\mathbb{R}^{n} \backslash A}\right\|_{1} .
$$

Hence

$$
\inf _{f_{1}+f_{2}=f}\left(\left\|f_{1}\right\|_{r^{\prime}(\cdot)}+t\left\|f_{2}\right\|_{1}\right) \geqslant \frac{1}{2} \inf _{A \subset \mathbb{R}^{n}}\left(\left\|f \chi_{A}\right\|_{r^{\prime}(\cdot)}+t\left\|f \chi_{\mathbb{R}^{n} \backslash A}\right\|_{1}\right) .
$$

On the other hand the opposite inequality holds with constant 1 , since we may choose $f_{1}=f \chi_{A}$ and $f_{2}=f \chi_{\mathbb{R}^{n} \backslash A}$ in the first infimum. So we conclude that

$$
\inf _{f_{1}+f_{2}=f}\left(\left\|f_{1}\right\|_{r^{\prime}(\cdot)}+t\left\|f_{2}\right\|_{1}\right) \approx \inf _{A \subset \mathbb{R}^{n}}\left(\left\|f \chi_{A}\right\|_{r^{\prime}(\cdot)}+t\left\|f \chi_{\mathbb{R}^{n} \backslash A}\right\|_{1}\right) .
$$

Since $r^{\prime}>1$, the infimum is not achieved when $\sup \left\{|f| \chi_{A}\right\}>\inf \left\{|f| \chi_{\mathbb{R}^{n} \backslash A}\right\}$ (since in this case we can shift mass to decrease the $L^{r^{\prime}(\cdot)}$-norm while conserving the $L^{1}$-norm). Assuming that $|\{f=c\}|=0$ for all $c \in \mathbb{R}$, it follows that $A$ must be of the form $\{|f|<c\}$ for some $c \geqslant 0$. In our case, $f$ is radially decreasing and so $A=\mathbb{R}^{n} \backslash B(R)$, for some $R \in[\delta, \infty]$. This corresponds to the functions $f_{1}=|x-\cdot|^{\alpha-n} \chi_{\mathbb{R}^{n} \backslash B(R)}$ and $f_{2}=|x-\cdot|^{\alpha-n} \chi_{B(R) \backslash B}$.

For simplicity we denote $s:=r_{\alpha}^{\#}$. A straight calculation gives $\left\|f_{2}\right\|_{1} \approx$ $R^{\alpha}-\delta^{\alpha}$. Then it follows from Lemma 6.1 that

$$
\|f\|_{\left(L^{\left.r^{\prime}(\cdot), L^{1}\right)_{\theta, 1}}\right.} \approx \int_{0}^{\infty} t^{-\theta} \inf _{R \in[\delta, \infty)}\left(R^{-\frac{n}{s_{B(R)}}}+t\left(R^{\alpha}-\delta^{\alpha}\right)\right) \frac{d t}{t} .
$$


By [9, Corollary 4.5.9], $R^{-\frac{n}{s_{B(R)}}} \approx R^{-\frac{n}{q}}$ where $q:=s_{\infty}$ if $R \geqslant 1$ and $q:=s(x)$ otherwise. Recall that $s_{\infty}$ is the limit value of $s$ at infinity, from the definition of log-Hölder continuity.

We further observe that

$$
\inf _{R \in[\delta, \infty)}\left(R^{-\frac{n}{q}}+t\left(R^{\alpha}-\delta^{\alpha}\right)\right) \approx \inf _{R \in[\delta, \infty)}\left(\frac{\alpha q}{n} R^{-\frac{n}{q}}+t\left(R^{\alpha}-\delta^{\alpha}\right)\right),
$$

since $\frac{\alpha q}{n}$ is bounded away from 0 and infinity. Then we apply Lemma 6.2 twice, for $\beta=\frac{n}{s_{\infty}}$ and $\beta=\frac{n}{s(x)}$, to conclude that

$$
\inf _{R \in[\delta, \infty]}\left(\frac{\alpha q}{n} R^{-\frac{n}{q}}+t\left(R^{\alpha}-\delta^{\alpha}\right)\right) \approx \min \left\{t^{\frac{n}{n+\alpha q}}, \delta^{-\frac{n}{q}}\right\},
$$

where $q:=s_{\infty}$ if and only if $t \geqslant 1$ and $\delta \leqslant 1$ and $q:=s(x)$ otherwise.

Let $t_{0}>0$ be such that $t_{0}^{\frac{n}{n+\alpha q}}=\delta^{-\frac{n}{s_{B}}}$. Then

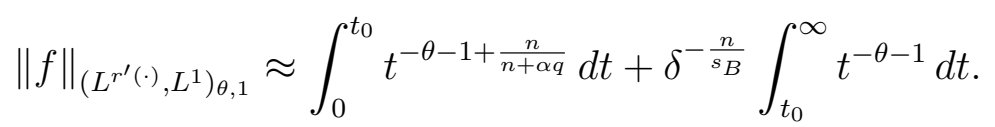

If $t_{0}>1$ (so that $\delta<1$ ) we find that

$$
\int_{0}^{t_{0}} t^{-\theta-1+\frac{n}{n+\alpha q}} d t=\int_{0}^{1} t^{-\theta-1+\frac{n}{n+\alpha s \infty}} d t+\int_{1}^{t_{0}} t^{-\theta-1+\frac{n}{n+\alpha s(x)}} d t \approx t_{0}^{-\theta+\frac{n}{n+\alpha s(x)}} .
$$

So in this case

$$
\|f\|_{\left(L^{\left.r^{\prime}(\cdot), L^{1}\right)_{\theta, 1}}\right.} \approx\left(t_{0}^{-\theta+\frac{n}{n+\alpha s(x)}}+\delta^{-\frac{n}{s_{B}}} t_{0}^{-\theta}\right) \approx \delta^{-\frac{n}{s_{B}}+\theta \frac{n}{s_{B}} \frac{n+\alpha s(x)}{n}} .
$$

Since $p$ is log-Hölder continuous and $x \in B=B(x, \delta)$, we have $\delta^{s_{B}} \approx$ $\delta^{s(x)}$. Thus

$$
\delta^{-\frac{n}{s_{B}}+\theta \frac{n}{s_{B}} \frac{n+\alpha s(x)}{n}}=\delta^{(\theta \alpha s(x)+(\theta-1) n) \frac{1}{s_{B}}} \approx \delta^{\theta \alpha+(\theta-1) n \frac{1}{s_{B}}}=\delta^{-\frac{n}{p_{B}^{\#}}} .
$$

For $t_{0} \leqslant 1$ we similarly conclude that $\|f\|_{\left(L^{\left.r^{\prime}(\cdot), L^{1}\right)_{\theta, 1}}\right.} \approx \delta^{-\frac{n}{p_{B}^{\#}}}$, using that $\delta^{s_{B}} \approx \delta^{s_{\infty}}$ which holds by the log-Hölder decay since $\delta \geqslant 1$.

According to [29, Theorem 1.11.2] the duality formula

$$
\left(\left(A_{0}, A_{1}\right)_{\theta, q}\right)^{*}=\left(A_{0}^{*}, A_{1}^{*}\right)_{\theta, q^{\prime}}
$$

holds when $q \in[1, \infty)$ and $A_{0} \cap A_{1}$ is dense both in $A_{0}$ and in $A_{1}$. We choose $A_{0}=L^{p^{\prime}(\cdot)}\left(\mathbb{R}^{n}\right), A_{1}=L^{1}\left(\mathbb{R}^{n}\right)$ and $q=1$. Then we obtain

$$
\left(L^{p^{\prime}(\cdot)}, L^{1}\right)_{\theta, 1}^{*}=\left(L^{p(\cdot)}, L^{\infty}\right)_{\theta, \infty} .
$$

Hence we obtain the Hölder inequality

$$
\int_{\mathbb{R}^{n}} f(x) g(x) d x \lesssim\|f\|_{\left(L^{p(\cdot)}, L^{\infty}\right)_{\theta, \infty}}\|g\|_{\left(L^{\left.p^{\prime}(\cdot), L^{1}\right)_{\theta, 1}}\right.} .
$$

In the following result we generalize [9, Lemma 6.1.5] where the same conclusion was reached under the stronger assumption that $\|f\|_{L^{p(\cdot)}} \leqslant 1$. 
Lemma 6.4. Let $p \in \mathcal{P}_{1}^{\log }\left(\mathbb{R}^{n}\right)$ with $1<p^{-} \leqslant p^{+}<\frac{n}{\alpha}$ for $\alpha \in(0, n)$. Let $x \in \mathbb{R}^{n}, \delta>0$, and $f \in w-L^{p(\cdot)}\left(\mathbb{R}^{n}\right)$ with $\|f\|_{w-L^{p(\cdot)}} \leqslant 1$. Then

$$
\int_{\mathbb{R}^{n} \backslash B(x, \delta)} \frac{|f(y)|}{|x-y|^{n-\alpha}} d y \lesssim|B(x, \delta)|^{-\frac{1}{\left(p_{\alpha}^{\#}\right)_{B(x, \delta)}}} .
$$

Proof. Set $B:=B(x, \delta)$ and $r:=(1-\theta) p$, where $\theta>0$ is so small that $r^{-}>1$. By Theorem 5.1 we have $\left(L^{r(\cdot)}, L^{\infty}\right)_{\theta, \infty}=w-L^{p(\cdot)}$ and thus by Hölder's inequality, the assumption $\|f\|_{w-L^{p(\cdot)}} \leqslant 1$ and Lemma 6.3 we obtain that

$\int_{\mathbb{R}^{n} \backslash B} \frac{|f(y)|}{|x-y|^{n-\alpha}} d y \lesssim\|f\|_{\left(L^{r(\cdot)}, L^{\infty}\right)_{\theta, \infty}}\left\||x-\cdot|^{\alpha-n}\right\|_{\left(L^{\left.r^{\prime}(\cdot), L^{1}\right)_{\theta, 1}}\right.} \lesssim|B|^{-\frac{1}{\left(p_{\alpha}^{\#}\right)_{B}}}$.

With this result we immediately obtain a generalization of [9, Lemma 6.1.8] as follows, where similarly the condition $\|f\|_{p(\cdot)} \leqslant 1$ has been replaced by $\|f\|_{w-L^{p}(\cdot)} \leqslant 1$ :

Lemma 6.5. Let $p \in \mathcal{P}_{1}^{\log }\left(\mathbb{R}^{n}\right)$ with $1<p^{-} \leqslant p^{+}<\frac{n}{\alpha}$ for $\alpha \in(0, n)$. Then

$$
\mathcal{I}_{\alpha} f(x)^{p_{\alpha}^{\#}(x)} \lesssim \mathcal{M} f(x)^{p(x)}+h(x),
$$

for all $x \in \mathbb{R}^{n}$, and $f \in w-L^{p(\cdot)}\left(\mathbb{R}^{n}\right)$ with $\|f\|_{w-L^{p(\cdot)}} \leqslant 1$, where $h \in$ $w-L^{1}\left(\mathbb{R}^{n}\right) \cap L^{\infty}\left(\mathbb{R}^{n}\right)$ is positive. The implicit constant and $h$ depend only on log-Hölder constant of $p, p^{-}, p^{+}, \alpha$, and $n$.

Then we obtain the following analogue of [9, Theorem 6.1.9] using the previous lemma and Theorem 1.3:

Theorem 6.6. Let $p \in \mathcal{P}_{1}^{\log }\left(\mathbb{R}^{n}\right), \alpha \in \mathcal{P}_{0}^{\log }\left(\mathbb{R}^{n}\right)$ and $1<p^{-} \leqslant p^{+}<\frac{n}{\alpha^{+}}$. If $f \in w-L^{p(\cdot)}\left(\mathbb{R}^{n}\right)$ and $|f|^{p(\cdot) / p_{\alpha}^{\#}(\cdot)} \in w-L^{p_{\alpha}^{\#}(\cdot)}\left(\mathbb{R}^{n}\right)$, then the function $x \mapsto$ $\mathcal{I}_{\alpha(x)} f(x)$ belongs to $w-L^{p_{\alpha}^{\#}(\cdot)}\left(\mathbb{R}^{n}\right)$.

Proof. We write $t:=p / p_{\alpha}^{\#}$. By a scaling argument we may assume that $\|f\|_{w-L^{p}(\cdot)} \leqslant 1$. By Lemma 6.5, there exists $h \in w-L^{1}\left(\mathbb{R}^{n}\right) \cap L^{\infty}\left(\mathbb{R}^{n}\right)$ such that $\left(\mathcal{I}_{\alpha(x)} f(x)\right)^{p_{\alpha}^{\#}(x)} \leqslant c\left(\mathcal{M} f(x)^{p(x)}+h(x)\right)$. Then

$$
\left\{\mathcal{I}_{\alpha(x)} f(x)>\lambda\right\} \subset\left\{\mathcal{M} f(x)^{t(x)}>c \lambda\right\} \cup\left\{h^{1 / p_{\alpha}^{\#}(x)}>c \lambda\right\}
$$

and so we obtain

$$
\int_{\left\{\mathcal{I}_{\alpha(x)} f(x)>\lambda\right\}} \lambda^{p_{\alpha}^{\#}(x)} d x \leqslant \int_{\left\{\mathcal{M} f(x)^{t(x)}>c \lambda\right\}} \lambda^{p_{\alpha}^{\#}(x)} d x+\int_{\left\{h^{\left.1 / p_{\alpha}^{*}(x)>c \lambda\right\}}\right.} \lambda^{p_{\alpha}^{\#}(x)} d x .
$$

Now $h \in w-L^{1}\left(\mathbb{R}^{n}\right) \cap L^{\infty}\left(\mathbb{R}^{n}\right) \subset L^{p_{\alpha}^{\#}(\cdot)}\left(\mathbb{R}^{n}\right)$, so the last term is bounded. Thus it remains to show that $(\mathcal{M} f)^{t(\cdot)} \in w-L^{p_{\alpha}^{\#}(\cdot)}\left(\mathbb{R}^{n}\right)$.

Let $t_{0} \in\left(1 /\left(p_{\alpha}^{\#}\right)^{-}, t^{-}\right)$. Since $|f|^{t(\cdot)} \in w-L^{p_{\alpha}^{\#}(\cdot)}\left(\mathbb{R}^{n}\right)$, we obtain that $|f|^{t(\cdot) / t_{0}} \in w-L^{t_{0} p_{\alpha}^{\#}(\cdot)}\left(\mathbb{R}^{n}\right)$. By assumption, $\left(t_{0} p_{\alpha}^{\#}\right)^{-}>1$ and hence it follows from Theorem 1.3 that $\mathcal{M}\left(|f|^{t(\cdot) / t_{0}}\right) \in w-L^{t_{0} p_{\alpha}^{\#}(\cdot)}\left(\mathbb{R}^{n}\right)$. Since $t / t_{0} \geqslant$ 
1, Lemma 4.2 implies that $(\mathcal{M} f)^{t(\cdot) / t_{0}} \in w-L^{t_{0} p_{\alpha}^{\#}(\cdot)}\left(\mathbb{R}^{n}\right)$, and thus $(\mathcal{M} f)^{t(\cdot)} \in$ $w-L^{p_{\alpha}^{\#}(\cdot)}\left(\mathbb{R}^{n}\right)$.

As was noted before, $\mathcal{I}_{\alpha(x)} f(x) \approx \mathcal{I}_{\alpha(\cdot)} f(x)$ in bounded domains. Furthermore, a log-Hölder continuous exponent in a domain can be extended to a variable exponent in the whole space, with the same parameters [9, Proposition 4.1.7]. Thus we obtain the following corollary.

Corollary 6.7. Let $p \in \mathcal{P}_{1}^{\log }(\Omega), \alpha \in \mathcal{P}_{0}^{\log }(\Omega), p^{-}>1$ and $(\alpha p)^{+}<n$. If $f \in w-L^{p(\cdot)}(\Omega)$ and $|f|^{p(\cdot) / p_{\alpha}^{\#}(\cdot)} \in w-L^{p_{\alpha(\cdot)}^{\#}}(\Omega)$, then $\mathcal{I}_{\alpha(\cdot)} f \in w-L^{p_{\alpha}^{\#}(\cdot)}(\Omega)$.

Note that a direct use of Theorem 6.6 leads to the assumption $\alpha^{+} p^{+}<n$ in the corollary. However, $(\alpha p)^{+}<n$ if and only if the domain can be split into a finite number of parts in each of which the inequality $\alpha^{+} p^{+}<n$ holds, so in fact these conditions are equivalent.

\section{The WolfF POTENTIAL}

Let $\mu$ be a positive, locally finite Borel measure. The (truncated) Wolff potential is defined by

$$
\mathcal{W}_{\alpha, p}^{\mu}(x, R):=\int_{0}^{R}\left(\frac{\mu(B(x, r))}{r^{n-\alpha p}}\right)^{1 /(p-1)} \frac{d r}{r}
$$

with the full Wolff potential being $\mathcal{W}_{\alpha, p}^{\mu}(x):=\mathcal{W}_{\alpha, p}^{\mu}(x, \infty)$. There are several ways in which this can be generalized to the variable exponent setting. The most straigth-forward is to consider the point-wise potential $x \mapsto \mathcal{W}_{\alpha(x), p(x)}^{\mu}(x, R)$.

In this case we immediately obtain the following inequality from the constant exponent setting:

$$
\mathcal{W}_{\alpha(x), p(x)}^{\mu}(x) \lesssim \mathcal{I}_{\alpha(x)}\left(\mathcal{I}_{\alpha(x)} \mu^{\frac{1}{p(x)-1}}\right)(x) .
$$

This was observed in [6, Subsection 5.2]. As we have noted, in the bounded domain case the Riesz potentials $\mathcal{I}_{\alpha(x)} f(x)$ and $\mathcal{I}_{\alpha(\cdot)} f(x)$ are comparable. Thus we obtain that

$$
\mathcal{W}_{\alpha(x), p(x)}^{\mu}(x, R) \lesssim \mathcal{I}_{\alpha(\cdot)}\left(\mathcal{I}_{\alpha(\cdot)} \mu^{\frac{1}{p(x)-1}}\right)(x) .
$$

However, there is no immediate way to change the exponent $\frac{1}{p(x)-1}$. As far as we can see, the above inequality cannot be used to derive Theorem 8.6, thus the validity of the claims in this part of [6, Section 5.2] are in doubt. (Additionally, their claim that $\mathcal{I}_{\alpha(\cdot)}: L^{p(\cdot)}\left(\mathbb{R}^{n}\right) \rightarrow L^{p_{\alpha}^{\#}(\cdot)}\left(\mathbb{R}^{n}\right)$ is bounded is false, see [16, Example 4.1]; the claim only holds for bounded domains. Of course, the latter claim is what is actually needed.)

The Wolff potential has also been studied by F.-Y. Maeda [24]. To state the result as clearly as possible, let us denote $g(y):=\mathcal{I}_{\alpha} f(y)^{\frac{1}{p(y)-1}}$. Maeda proved that

$$
\mathcal{W}_{\alpha, p(x)}^{\mu}(x) \lesssim \mathcal{I}_{\alpha} g(x)
$$


Since $\mathcal{I}_{\alpha(x)} f(x) \approx \mathcal{I}_{\alpha(\cdot)} f(x)$, this implies the desired inequality, which can succinctly be stated as

$$
\mathcal{W}_{\alpha(x), p(x)}^{\mu}(x) \lesssim \mathcal{I}_{\alpha(\cdot)}\left(\mathcal{I}_{\alpha(\cdot)} \mu^{\frac{1}{p(\cdot)-1}}\right)(x)
$$

provided one keeps track of which dot is related to which operation. The right hand side in this equation is called the Havin-Maz'ya potential which is denoted by $\mathcal{V}_{\alpha(\cdot), p(\cdot)}^{\mu}(x)$.

The following result is now a consequence of Theorems 4.3 and 6.6, and (7.1).

Theorem 7.2. Let $\alpha, r$, and $p$ be bounded and log-Hölder continuous, with $p^{-}>1$ and $r^{-} \geqslant 1,0<\alpha^{-} \leqslant \alpha^{+}<n$, $(\alpha p r)^{+}<n$, and $p(x) \geqslant$ $1+1 / r(x)-\alpha(x) / n$ for every $x \in \Omega$. If $f \in L^{r(\cdot)}(\Omega)$, then

$$
x \mapsto \mathcal{W}_{\alpha(x), p(x)}^{f}(x) \in w-L^{\frac{n r(\cdot)(p(\cdot)-1)}{n-\alpha(\cdot) p(\cdot) r(\cdot)}}(\Omega) .
$$

Proof. By (7.1), it suffices to consider the Havin-Maz'ya potential $\mathcal{V}_{\alpha(\cdot), p(\cdot)}^{f}$ instead of the Wolff potential. Denote $s:=\frac{n r(p-1)}{n-\alpha r}$; by assumption $p \geqslant$ $1+1 / r-\alpha / n$ so that $s^{-} \geqslant 1$. Choosing $q:=1 /(p-1)$ in Theorem 4.3, we see that

$$
\left(\mathcal{I}_{\alpha(\cdot)} f\right)^{1 /(p(\cdot)-1)} \in w-L^{s(\cdot)}(\Omega)
$$

Since $(\alpha p r)^{+}<n$, we find that $(\alpha s)^{+}<n$ and thus by choosing $q:=$ $s /\left[(p-1) s_{\alpha}^{\#}\right]$ in Theorem 4.3 we obtain that

$$
\left[\left(\mathcal{I}_{\alpha(\cdot)} f\right)^{1 /(p(\cdot)-1)}\right]^{\frac{s(\cdot)}{s_{\alpha}^{\#}(\cdot)}} \in w-L^{\frac{(p(\cdot)-1) s_{\alpha}^{\#}(\cdot) \frac{n r(\cdot)}{n-\alpha(\cdot) r(\cdot)}}{s(\cdot)}}(\Omega)=w-L^{s_{\alpha}^{\#}(\cdot)}(\Omega) .
$$

Further, since $(\alpha p r)^{+}<n$, we can use Corollary 6.7 for the function $\left(\mathcal{I}_{\alpha(\cdot)} f\right)^{1 /(p(\cdot)-1)}$ to conclude that

$$
\mathcal{V}_{\alpha(\cdot), p(\cdot)}^{f} \in w-L^{s_{\alpha}^{\#}(\cdot)}(\Omega) .
$$

The claim follows from this since $s_{\alpha}^{\#}=\frac{n r(p-1)}{n-\alpha p r}$.

\section{AN APPLICATION TO PARTIAL DIFFERENTIAL EQUATIONS}

In this section, we discuss consequences of our results and pointwise potential estimates for solutions to the nonlinear elliptic equation

$$
-\operatorname{div}\left(|\nabla u|^{p(x)-2} \nabla u\right)=\mu,
$$

where $\mu$ is a Borel measure with finite mass. The right quantity for estimating solutions to (8.1) and their gradients is the Wolff potential $\mathcal{W}_{\alpha(x), p(x)}^{\mu}(x)$.

Recall that for right hand side data a Borel measure $\mu$ with finite mass or a function in $L^{1}$, we use the notion of solutions obtained as limits of approximations, SOLAs for short. Gradient potential estimates for SOLAs follow by working with a priori more regular solutions, and then transferring the information obtained to the limit. In the case of general measures, the latter step requires some care, as the approximants converge only in the sense of 
weak convergence of measures. For this reason, the approximation argument is not done using the final potential estimates. Certain intermediate estimates, from which the actual potential estimates are then built, need to be used instead. See [6, Proof of Theorem 1.4, p. 668] for details.

An alternative point of view is to start with the fundamental objects of the nonlinear potential theory related to the $p(\cdot)$-Laplacian, namely $p(\cdot)$ superharmonic functions. See [23, Definition 2.1, p. 1068] for the exact definition of this class. For a $p(\cdot)$-superharmonic function $u$, there exists a measure $\mu$ such that (8.1) holds. This is the Riesz measure of $u$. Important results in nonlinear potential theory are derived by employing measure data equations like (8.1). The leading example is the necessity of the celebrated Wiener criterion for boundary regularity, see [19].

The gradient potential estimates in [6] are local: one works in a fixed ball, compactly contained in $\Omega$. Thus the solution under consideration can be a local SOLA, i.e. it suffices to choose approximations in a fixed compact subset of $\Omega$.

If $\mu$ is a signed measure, we use the notation

$$
\mathcal{W}_{\alpha(x), p(x)}^{\mu}(x, R)=\int_{0}^{R}\left(\frac{|\mu|(B(x, r))}{r^{n-\alpha(x) p(x)}}\right)^{1 /(p(x)-1)} \frac{\mathrm{d} r}{r},
$$

where $|\mu|$ is the total variation of $\mu$.

To extend the gradient potential estimate to $p(\cdot)$-superharmonic functions, we need the fact that these functions are local SOLAs. This is the content of the following theorem.

Theorem 8.2. Let $p$ be log-Hölder continuous with $p^{-} \geqslant 2$. Let $u$ be a $p(\cdot)$-superharmonic function in a domain $\Omega$ and let $\mu$ be the measure such that

$$
-\operatorname{div}\left(|\nabla u|^{p(x)-2} \nabla u\right)=\mu .
$$

For every subdomain $\Omega^{\prime} \Subset \Omega$ there are sequences of solutions $\left(u_{i}\right)$ and smooth, positive functions $\left(f_{i}\right)$ such that

$$
-\operatorname{div}\left(\left|\nabla u_{i}\right|^{p(x)-2} \nabla u_{i}\right)=f_{i} \text { in } \Omega^{\prime},
$$

$u_{i} \rightarrow u$ in $W^{1, q(\cdot)}\left(\Omega^{\prime}\right)$ for any continuous $q$ such that $q(x)<\frac{n}{n-1}(p(x)-1)$ for all $x \in \overline{\Omega^{\prime}}$, and $f_{i} \rightarrow \mu$ in the sense of weak convergence of measures.

Proof. This follows in the same way as in the constant exponent case, Theorem 2.7 in [20]. For the reader's convenience, we sketch the argument here with the appropriate references for various auxiliary results. The proof consists of two main steps. First, we prove the claim when $u$ is a weak supersolution. The general case is then reduced to the case of supersolutions by an approximation argument using the obstacle problem.

Assume first that $u$ is a weak supersolution. Then $u \in W_{\text {loc }}^{1, p(\cdot)}(\Omega)$, and the fact that $\mu$ belongs to the dual space $\left(W_{0}^{1, p(\cdot)}\left(\Omega^{\prime}\right)\right)^{*}$ follows from the equation satisfied by $u$. Then the case of supersolutions follows by arguing 
as in [20, Lemma 2.6] and using the elementary inequalities between the $p$-modular and the Luxemburg norm.

In the general case, the fact that $u \in W^{1, q(\cdot)}\left(\Omega^{\prime}\right)$ follows by a refinement of [22, Theorem 4.4]. By [13, Theorem 6.5], we may choose a sequence $\left(\widetilde{u}_{i}\right)$ of continuous weak supersolutions increasing to $u$. Arguing as in [13, proof of Theorem 5.1] we can show that $\nabla \min \left(\widetilde{u}_{i}, k\right) \rightarrow \nabla \min (u, k)$ pointwise almost everywhere for any $k \in \mathbb{R}$. It follows that $\nabla \widetilde{u}_{i} \rightarrow \nabla u$ pointwise a.e., and the pointwise convergences easily imply that $\widetilde{u}_{i} \rightarrow u$ in $W^{1, q(\cdot)}\left(\Omega^{\prime}\right)$. The proof is completed by applying the case of supersolutions to the functions $\widetilde{u}_{i}$, together with the convergence of $\widetilde{u}_{i} \rightarrow u$ in $W^{1, q(\cdot)}\left(\Omega^{\prime}\right)$, see the proof of Theorem 2.7 in [20].

The following pointwise potential estimates hold for local SOLAs and $p(\cdot)$-superharmonic functions. See [6] for (8.4) and (8.5) in the case of SOLAs, and [23] for (8.4) for $p(\cdot)$-superharmonic functions. Finally, the gradient estimate $(8.5)$ holds also for $p(\cdot)$-superharmonic functions by an application of Theorem 8.2. The Hölder continuity of $p$ is required for the gradient estimate, since its proof uses Hölder estimates for the gradient of weak solutions (cf. [1]).

Theorem 8.3. Let $p$ be log-Hölder continuous with $p^{-} \geqslant 2$. Let $u$ be positive $p(\cdot)$-superharmonic or a local SOLA to (8.1). Then there exists $\gamma>0$ such that

$$
\left|u\left(x_{0}\right)\right| \lesssim\left(f_{B\left(x_{0}, R\right)}|u|^{\gamma} d x\right)^{1 / \gamma}+\mathcal{W}_{1, p\left(x_{0}\right)}^{\mu}\left(x_{0}, 2 R\right)+R
$$

for all sufficiently small $R>0$. For positive $p(\cdot)$-superharmonic functions, the assumption $p^{-}>1$ suffices instead of $p^{-} \geqslant 2$.

Suppose next that $p$ is Hölder continuous. Then

$$
\left|\nabla u\left(x_{0}\right)\right| \lesssim f_{B\left(x_{0}, R\right)}|\nabla u| d x+\mathcal{W}_{1 / p\left(x_{0}\right), p\left(x_{0}\right)}^{\mu}\left(x_{0}, 2 R\right)+R
$$

for all sufficiently small $R>0$.

The restriction $p^{-} \geqslant 2$ in the gradient estimates is related to the fact that there are substantial differences in gradient potential estimates in the cases $p<2$ and $p>2$ even with constant exponents, see [11]. For simplicity, we focus on the prototype case (8.1) here, but this result, and hence also Theorem 8.6 below, hold for more general equations of the form

$$
-\operatorname{div}(a(x, \nabla u))=\mu
$$

under appropriate structural assumptions on $a(x, \xi)$. The interested reader may refer to $[6,23]$ for details.

The following result is an immediate consequence of Theorems 7.2 and 8.3. 
Theorem 8.6. Let $p$ and $r$ be log-Hölder continuous with $p^{-} \geqslant 2$. Let $u$ be a positive $p(\cdot)$-superharmonic function, or a local SOLA to (8.1), with $\mu \in L^{r(\cdot)}(\Omega)$.

(a) If $(p r)^{+}<n$ and $p \geqslant 1+1 / r-1 / n$ for every $x \in \Omega$, then

$$
u \in w-L_{\mathrm{loc}}^{\frac{n r(\cdot)(p(\cdot)-1)}{n-(\cdot)(\cdot)}}(\Omega) .
$$

For positive $p(\cdot)$-superharmonic functions, the assumption $p^{-}>1$ suffices.

(b) Suppose in addition that $p$ is Hölder continuous. If $r^{+}<n$ and $p \geqslant 1+1 / r-1 /(n p)$ for every $x \in \Omega$, then

$$
|\nabla u| \in w-L_{\mathrm{loc}}^{\frac{n r(\cdot)(p(\cdot)-1)}{n-r(\cdot)}}(\Omega) .
$$

If $r \equiv 1, \mu$ can be a measure with finite mass instead of a function. Each of the inclusions comes with an explicit estimate.

Theorem 1.5 is of course contained in the above theorem when $r \equiv 1$. The interesting case in these results is when $r^{-}=1$; if $r^{-}>1$, we can use the pointwise inequality (7.1) and the strong-to-strong estimate for the Riesz potential to get estimates in strong Lebesgue spaces with the same exponents.

When $r \equiv 1$, the above inclusions are sharp for constant $p$ on the scale of $w-L^{q}$ spaces. This is a special case of the following examples.

Example 8.7. Let $B$ be the unit ball in $\mathbb{R}^{n}$, and assume that the exponent $p$ is smooth and radial. Define the function $u$ by

$$
u(x):=\int_{|x|}^{1}\left(p(\varrho) \varrho^{n-1}\right)^{-1 /(p(\varrho)-1)} \mathrm{d} \varrho .
$$

Then by [13, Section 6] $u$ is $p(\cdot)$-superharmonic in $B$, and Theorem 4.10 of [22] implies that

$$
-\operatorname{div}\left(|\nabla u|^{p(x)-2} \nabla u\right)=K \delta,
$$

where $K>0$ and $\delta$ is Dirac's delta at the origin. The exact value of $K$ is not important.

Assume that $q$ is log-Hölder continuous. We will show that $u \in w-L^{q(\cdot)}(B)$ if and only if

$$
q(0) \leqslant \frac{n(p(0)-1)}{n-p(0)}
$$

and $|\nabla u| \in w-L^{q(\cdot)}(B)$ if and only if

$$
q(0) \leqslant \frac{n(p(0)-1)}{n-1} .
$$

We reason as follows to get these characterizations. First, log-Hölder continuity of $p$ implies that

$$
|u(x)| \approx|x|^{-\frac{n-p(0)}{p(0)-1}}
$$


and

$$
|\nabla u(x)| \approx|x|^{-\frac{n-1}{p(0)-1}}
$$

The inclusions

$$
\left\{t<c^{-1}|x|^{-\frac{n-p(0)}{p(0)-1}}\right\} \subset\{t<u(x)\} \subset\left\{t<c|x|^{-\frac{n-p(0)}{p(0)-1}}\right\}
$$

follow from (8.11), $c \geqslant 1$ being the constant implicit in (8.11).

We use the second inclusion in (8.13) to get

$$
\int_{\{u>t\}} t^{q(x)} \mathrm{d} x \leqslant \int_{\left\{t<c|x|^{-\frac{n-p(0)}{p(0)-1}}\right\}} t^{q(x)} \mathrm{d} x .
$$

We use the change of variables

$$
\lambda=t^{-\frac{p(0)-1}{n-p(0)}}
$$

and obtain that

$\int_{\left\{t<c|x|^{-\frac{n-p(0)}{p(0)-1}}\right\}} t^{q(x)} \mathrm{d} x \leqslant c \int_{\{|x|<\lambda\}} \lambda^{-q(x) \frac{n-p(0)}{p(0)-1}} \mathrm{~d} x \leqslant c \int_{\{|x|<\lambda\}} \lambda^{-q(0) \frac{n-p(0)}{p(0)-1}} \mathrm{~d} x$

where the last estimate follows from the log-Hölder continuity of $q$.

The last integral is finite if (8.9) holds. Starting from the first inclusion in (8.13), we get a similar lower bound. Hence $u \in w-L^{q(\cdot)}(B)$ if and only if (8.9) holds. Repeating the same argument using (8.12), we obtain the condition (8.10).

Example 8.14. The right hand side of the differential equation in the previous example is a delta measure, which is not an $L^{1}$-function. However, the example can be modified to yield a function in $L^{1}$. Denote by $u$ the function from the previous example and define

$$
v_{r}(x):= \begin{cases}a_{r}-b_{r}|x| & \text { when }|x| \leqslant r \\ u(x) & \text { otherwise. }\end{cases}
$$

The constants $a_{r}$ and $b_{r}$ are chosen so that $v_{r} \in C^{1}$. Then $a_{r}=|\nabla u(r)| \approx$ $r^{-\frac{n-1}{p(0)-1}}$ by the computations above. A direct calculation shows that

$$
-\operatorname{div}\left(\left|\nabla v_{r}\right|^{p(x)-2} \nabla v_{r}\right)=a_{r}^{p(x)-1}\left(\frac{n-1}{|x|}+p^{\prime}(x) \log a_{r}\right) .
$$

If we suppose that $p$ is Lipschitz continuous, then

$$
\frac{n-1}{|x|}+p^{\prime}(x) \log a_{r} \approx \frac{n-1}{|x|}
$$

for small enough $r$ and so the right hand side of this equation is positive in $B(0, r)$. Furthermore, the right hand side is in $L^{1}$ uniformly and $v_{r} \nearrow u$ as $r \rightarrow 0$, so we see that the conclusions from the previous example hold also for the $L^{1}$ case. 
Ackowledgments. We would like to thank the referee for useful comments which improved the readability of the paper.

A. Almeida was supported in part by FEDER funds through COMPETEOperational Programme Factors of Competitiveness ("Programa Operacional Factores de Competitividade") and by Portuguese funds through the Center for Research and Development in Mathematics and Applications (University of Aveiro) and the Portuguese Foundation for Science and Technology ("FCT-FundaÃ $\breve{g} \tilde{A}$ čo para a CiÃłncia e a Tecnologia"), within project PEstC/MAT/UI4106/2011 with COMPETE number FCOMP-01-0124-FEDER022690.

P. Hästö was supported in part by the Academy of Finland and the Swedish Cultural Foundation in Finland.

\section{REFERENCES}

[1] E. Acerbi and G. Mingione: Regularity results for a class of functionals with nonstandard growth, Arch. Ration. Mech. Anal. 156 (2001), no. 2, 121-140.

[2] A. Almeida and A. Caetano: Real interpolation of generalized Besov-Hardy spaces and applications, J. Fourier. Anal. Appl. 17 (2011), 691-719.

[3] A. Almeida and P. Hästö: Interpolation in variable exponent spaces, Rev. Mat. Complut., to appear.

[4] L. Boccardo and T. Gallouët: Nonlinear elliptic and parabolic equations involving measure data, J. Funct. Anal. 87 (1989), no. 1, 149-169.

[5] L. Boccardo and T. Gallouët: Nonlinear elliptic equations with right-hand side measures, Comm. Partial Differential Equations 17 (1992), no. 3-4, 641-655.

[6] V. Bögelein and J. Habermann: Gradient estimates via non standard potentials and continuity, Ann. Acad. Sci. Fenn. Math. 35 (2010), no. 2, 641-678.

[7] Yu. Brudnyı̆ and N. Krugljak: Interpolation Functors and Interpolation Spaces, Vol. 1, North-Holland, Amsterdam-New York-Oxford-Tokyo, 1991.

[8] L. Diening: Riesz potential and Sobolev embeddings of generalized Lebesgue and Sobolev spaces $L^{p(\cdot)}$ and $W^{k, p(\cdot)}$, Math. Nachr. 263 (2004), 31-43.

[9] L. Diening, P. Harjulehto, P. Hästö and M. Růžička: Lebesgue and Sobolev Spaces with Variable Exponents, Lecture Notes in Mathematics, 2017, Springer-Verlag, Heidelberg, 2011.

[10] L. Diening, P. Hästö and A. Nekvinda: Open problems in variable exponent Lebesgue and Sobolev spaces, FSDONA04 Proceedings (Drabek and Rákosník (eds.); Milovy, Czech Republic, 2004), 38-58.

[11] F. Duzaar and G. Mingione: Gradient estimates via linear and nonlinear potentials, $J$. Funct. Anal. 259 (2010), no. 11, 2961-2998.

[12] F. Duzaar and G. Mingione: Gradient estimates via non-linear potentials, Amer. J. Math. 133 (2011), no. 4, 1093-1149.

[13] P. Harjulehto, P. Hästö, M. Koskenoja, T. Lukkari and N. Marola: An obstacle problem and superharmonic functions with nonstandard growth, Nonlinear Anal. 67 (2007), no. 12, 3424-3440.

[14] P. Harjulehto, P. Hästö, U. Lê and M. Nuortio: Overview of differential equations with non-standard growth, Nonlinear Anal. 72 (2010), no. 12, 4551-4574.

[15] V. G. Maz'ya and V. P. Havin: A nonlinear potential theory, Russian Math. Surveys 27 (1972), no. 6, 71-148.

[16] P. A. Hästö: Local-to-global results in variable exponent spaces, Math. Res. Lett. 16 (2009), no. 2, 263-278. 
[17] H. Kempka and J. Vybíral: Lorentz spaces with variable exponents, Math. Nachr, to appear.

[18] T. Kilpeläinen and J. Malý: Degenerate elliptic equations with measure data and nonlinear potentials, Ann. Scuola Norm. Sup. Pisa Cl. Sci. (4) 19 (1992), no. 4, 591613.

[19] T. Kilpeläinen and J. Malý: The Wiener test and potential estimates for quasilinear elliptic equations, Acta Math. 172 (1994), no. 1, 137-161.

[20] J. Kinnunen, T. Lukkari, and M. Parviainen: Local approximation of superharmonic and superparabolic functions in nonlinear potential theory, J. Fixed Point Theory Appl. 13 (2013), no. 1, 291-307. .

[21] T. Lukkari: Elliptic equations with nonstandard growth involving measures, $\mathrm{Hi}$ roshima Math. J. 38 (2008), no. 1, 155-176.

[22] T. Lukkari: Singular solutions of elliptic equations with nonstandard growth, Math. Nachr. 282 (2009), no. 12, 1770-1787.

[23] T. Lukkari, F-Y. Maeda, and N. Marola: Wolff potential estimates for elliptic equations with nonstandard growth and applications, Forum Math. 22 (2010), no. 6, 10611087.

[24] F-Y. Maeda: Variable exponent version of Hedberg-Wolff inequalities, RIMS Kôkyûroku 1669 (2009), 68-80.

[25] G. Mingione: Gradient potential estimates, J. Eur. Math. Soc. (JEMS) 13 (2011), no. 2, 459-486.

[26] N. Samko, S. Samko and B. Vakulov: Weighted Sobolev theorem in Lebesgue spaces with variable exponent, J. Math. Anal. Appl. 335 (2007), no. 1, 560-583.

[27] S. Samko: Convolution and potential type operators in $L^{p(x)}\left(\mathbb{R}^{n}\right)$, Integral Transforms Spec. Funct. 7 (1998), no. 3-4, 261-284.

[28] M. Sanchón and J. M. Urbano: Entropy solutions for the $p(x)$-Laplace equation, Trans. Amer. Math. Soc. 361 (2009), no. 12, 6387-6405.

[29] H. Triebel: Interpolation theory, function spaces, differential operators, NorthHolland Mathematical Library, 18, North-Holland Publishing Co., Amsterdam-New York, 1978.

[30] H. Triebel: Theory of Function Spaces, Monographs in Mathematics 78, Birkhäuser Verlag, Basel, 1983.

A. Almeida

Center for Research and Development in Mathematics and Applications, Department of Mathematics, University of Aveiro, 3810-193 Aveiro, Portugal

jaralmeida@ua.pt

P. HARJULEHTO

Department of Mathematics and Statistics, FI-20014 University of Turku, Finland petteri.harjulehto@utu.fi

P. HÄSTÖ

Department of Mathematical Sciences, P.O. Box 3000, FI-90014 University of Oulu, Finland

peter.hasto@helsinki.fi

\section{T. LUKKARI}

Department of Mathematics and Statistics, P.O.Box 35 (MaD), FI-40014 University of Jyväskylä, Finland

teemu.j.lukkari@jyu.fi 